\title{
Non-Immune Hydrops Foetalis due to Congenital Toxoplasmosis: A Rare Case Report with Review of Literature.
}

\author{
Mallikarjun Adiveppa Pattanashetti ${ }^{1 *}$, Vijayalaxmi V Suranagi ${ }^{2}$ and Hema B Bannur ${ }^{2}$ \\ ${ }^{1}$ Department of Pathology, S. Nijalingappa Medical College , Bagalkot, India \\ ${ }^{2}$ Department of Pathology, Jawaharlal Nehru Medical College, Belagavi, India
}

\section{ABSTRACT}

Non-Immune Hydrops Foetalis (NIHF) implies an excess of total body water which manifests as extracellular accumulation of fluid in serous cavities and soft tissues of foetus without any detectable circulating antibody against RBC antigen. Toxoplasmosis is an important congenitally acquired infectious cause of NIHF. Mother had complaints of fever, reduced foetal movements, USG showed hydrops foetalis and she tested positive for Toxoplasma IgG antibodies. On autopsy, foetus had generalised subcutaneous oedema, bilateral hypoplastic lungs with pleural effusion and oedema of brain with ventriculomegaly suggestive of hydrocephalus. We report a rare case of NIHF due to congenital toxoplasmosis with bilateral hypoplastic lungs, with emphasis on epidemiology and prevention of toxoplasmosis.

Keywords: Non-Immune Hydrops Foetalis, Toxoplasmosis, Hypoplastic Lungs

\section{Introduction}

Non-Immune Hydrops Foetalis (NIHF) is an excess of total body water evident as extracellular accumulation of fluid in soft tissues and serous cavities of foetus without presence of identifiable circulating antibody against Red Blood Cell (RBC) antigen. Over 80 conditions are known to be associated with hydrops. ${ }^{[1]}$ One of the rare infectious causes of NIHF is Congenital toxoplasmosis. Seroprevalence of toxoplasmosis in reproductive age group Indian women is 2.9 to 42.5 percent. ${ }^{[2]}$ Global burden of congenital toxoplasmosis is 1.5 per 1000 live births. ${ }^{[3]} \mathrm{We}$ report a case of NIHF due to congenital toxoplasmosis with hydrocephalus and bilateral hypoplastic lungs for its rarity in India with emphasis on epidemiology and prevention of toxoplasmosis.

\section{Case Report}

A 24 year old mother with second gravida delivered a preterm 26 weeks intrauterine still born female foetus by breech extraction. Mother had complaints of fever in the first trimester for a week, reduced foetal movements in $2^{\text {nd }}$ trimester and on USG hydrops foetalis was noted. There was no history of congenital malformation, recurrent spontaneous abortion, bad obstetric outcome or hematological disorder in the family. No history of blood transfusion in the past. The mother was also a known case of $\mathrm{Rh}$ negative pregnancy with no Anti-D given after first delivery of healthy male baby weighing 2.5 $\mathrm{kg}$. Serological tests were performed for TORCH panel. Mother was positive for Toxoplasma IgG antibodies - 3.9 IU/L (Negative < 0.8 IU/ L ) and negative for Toxoplasma
IgM antibodies - $0.1 \mathrm{IU} / \mathrm{L}$ (Negative $<0.8 \mathrm{IU} / \mathrm{L}$ ) both done by ELISA method. She was negative for Rubella 0.7 IU/L (Negative < 0.8 IU/L ), CMV IgM - 0.05 IU/L (Negative $<0.8$ IU/L) and HSV $1 \& 2$ IgM assay - 0.04 IU/L ( Negative $<0.8$ IU/L). Mother was non- reactive for HBSAg and HIV virus.

On autopsy, foetus weighed 600 grams with generalised subcutaneous oedema of skin [Figure 1]. Facial dysmorphism in the form of low set ears and low hairline was seen. Foetus had hypoplasia of lungs with pleural effusion [Figure 2], hepatomegaly and ascites. Congestion and oedema of brain with ventriculomegaly suggestive of hydrocephalus was evident [Figure 3]. This case was diagnosed based on clinical history, autopsy examination of foetus and serological tests for toxoplasma gondii infection in mother.

\section{Discussion}

NIHF is the presence of $\geq 2$ abnormal fluid collections of foetus in the absence of red cell alloimmunization. It now accounts for almost $90 \%$ of cases of hydrops. ${ }^{[4]}$ The most common causes of NIHF are enlisted [Table 1]. ${ }^{[5]}$

Toxoplasma gondii, an opportunistic pathological intracellular parasite which is found in humans and other mammals causes serious zoonoses. In 1948, Sabin and Feldman discovered a serological test for the exploration of toxoplasma. The routes of transmission are ingestion of food contaminated by oocysts in cat faeces or undercooked meat of domestic animals and birds containing toxoplasma cysts, ingestion of raw or undercooked beef, lamb and pork, 


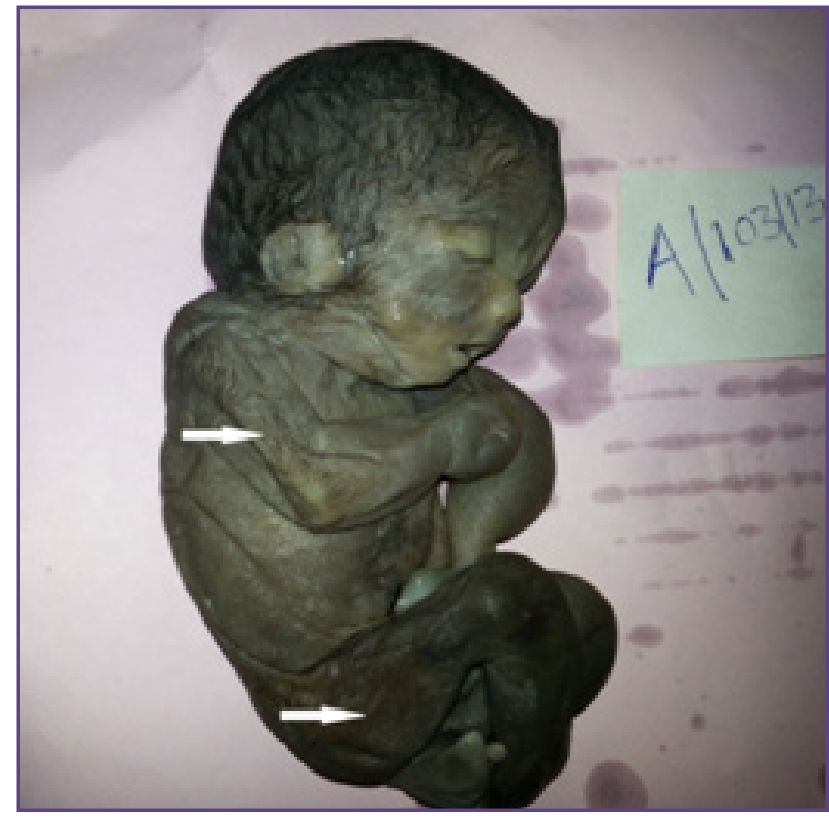

Fig. 1 : Generalised subcutaneous oedema of skin (Arrow).

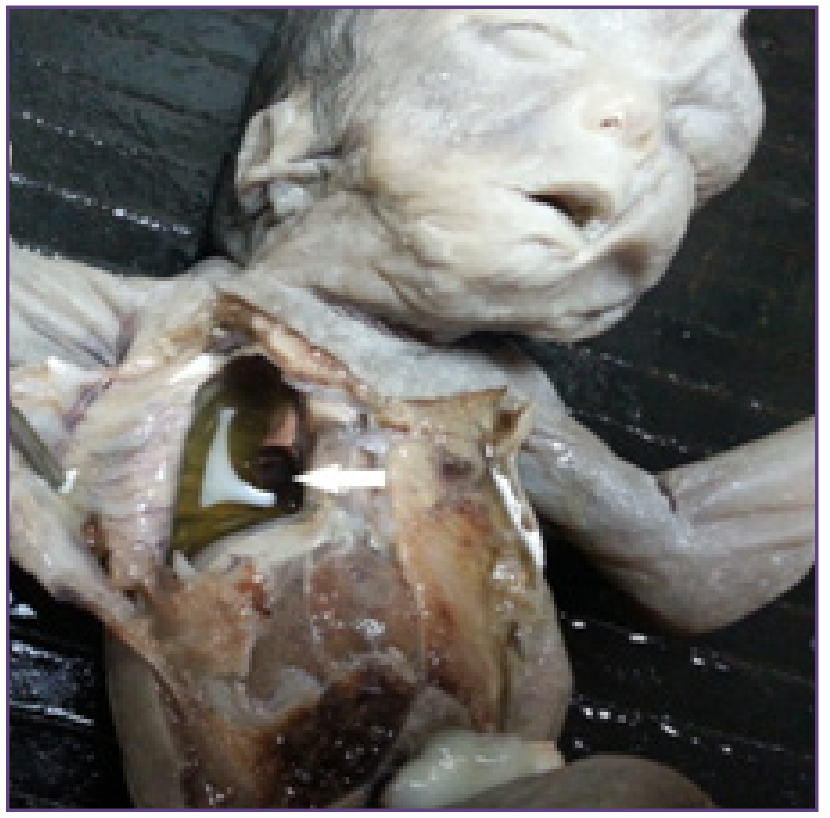

Fig. 2 : Foetus had hypoplasia of both lungs with pleural effusion (Arrow).

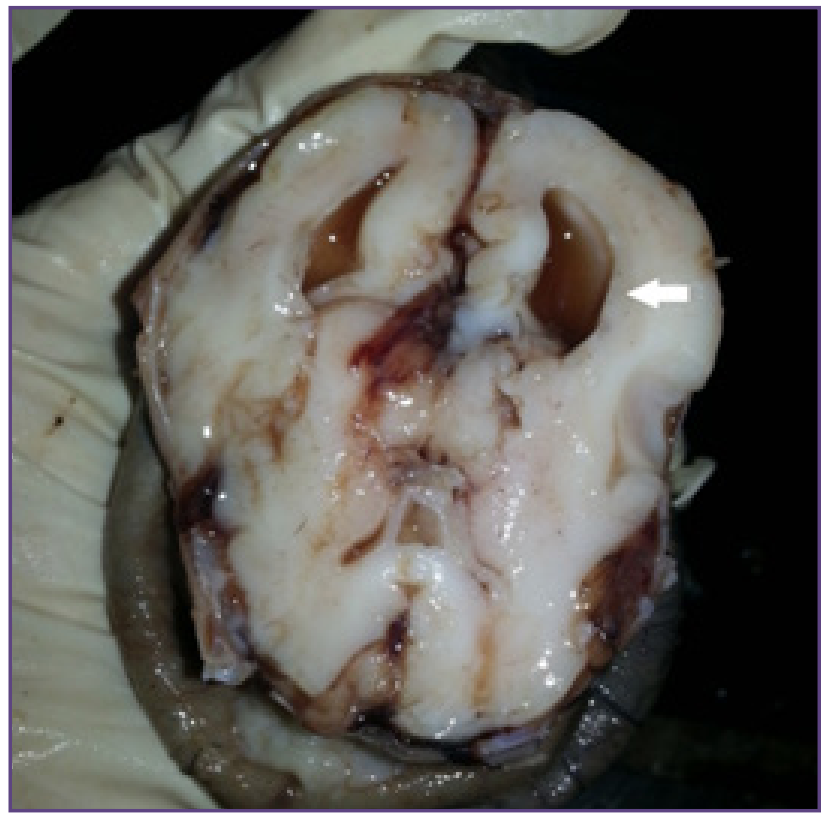

Fig. 3: Congestion and oedema of brain with ventriculomegaly suggestive of hydrocephalus (Arrow).

blood transfusion, consumption of unpasteurized goat's milk and contaminated drinking water, direct exposure to soil, injured skin and mucosa. ${ }^{[6]}$

Seroprevalence of toxoplasmosis in Indian women of child bearing age has remained a debatable topic as there are no published studies covering all the geographical regions. A seroprevalence study was undertaken by Singh $\mathrm{S}$ et al. ${ }^{[7]}$ on a large population of women of reproductive age from four distinct geographical regions of India : West, East, North and South India. This study showed an estimated total prevalence of $22.4 \%$. Among them, South India had highest prevalence $(37.3 \%)$ followed by East India $(21.2 \%)$, North India (19.7\%) and West India (8.8\%). The possible risk factors of infection in these women in their 
study included lower socioeconomic status, advanced age, consumption of raw salad, drinking untreated water, residing in mud plastered houses and owning pets. History of contact with animals was found in $29.1 \%$ women but pets were significantly more common in South Indian households. ${ }^{[7]}$ Association of sexual transmission of toxoplasmosis with multiple sexual exposures has also been reported in other studies. ${ }^{[8]}$

Several factors which favoured increased seroprevalence of toxoplasmosis in South India included climatic conditions, highly significant number of households with cats, socio-cultural factors wherein South Indians do not wear shoes and most often are barefoot or wear sleepers only, which increases chances of transferring oocysts from soil and water to their food. Dry arid climatic zone and high temperature of Western India was the cause of lower seroprevalence in that region. Water and food-borne outbreaks of toxoplasmosis have been are also reported worldwide and also from India. ${ }^{[9]}$

In pregnancy, mother-to-fetal transmission rates rise from $7 \%$ in the first trimester, to $24 \%$ in the second, to $59 \%$ in the third trimester. However, the incidence of severe fetal infection drops from $75 \%$ to a negligible risk in late pregnancy. ${ }^{[10]}$ Mother may be asymptomatic even with infection. Around 70-90\% of infants born with congenital infection do not have symptoms at birth. Visual impairment, mental retardation and learning disabilities generally manifest several months to years later. First and second trimester infection has been associated with spontaneous abortion, prematurity and death of the foetus. Clinical features of toxoplasmosis include chorioretinitis, blindness, strabismus, mental retardation, epilepsy, anemia, jaundice, rash, encephalitis, thrombocytopenia, pneumonitis, microcephaly, hypothermia diarrhea, intracranial calcification, hydrocephalus and nonspecific illness. Subclinical infection may be seen in $3^{\text {rd }}$ trimester. [11] The classic triad of chorioretinitis, intracranial calcifications and hydrocephalus is found in less than $10 \%$ of infants infected by toxoplasma. ${ }^{[12]}$

Congenital toxoplasmosis is a preventable infection. Primary prevention is possible by educating the pregnant women about the measures to prevent acquisition of toxoplasma infection. Prevention of primary infection depends upon educating pregnant women on the routes of transmission of Toxoplasma gondii and avoidance of high risk behaviors. Washing of fruits and vegetables should be done before eating. Washing of knives and the sink should be done after preparation of food. Consumption of unpasteurized milk, raw eggs or unfiltered water should be avoided. Measures such as cooking the meat at higher temperatures or keeping it frozen for one day are lethal to tachyzoites and bradyzoites of toxoplasma. Washing of hands with soap and water after working with soil, after touching raw or undercooked meat or after handling the cat is of utmost importance. ${ }^{[13]}$ Educational programs are a potentially powerful methods due to their low cost, and also because pregnant women are motivated to ensure the health of their babies. Doctors, nurses and health assistants can educate the pregnant women. Health education materials containing information about the prevention of T. gondii infection printed on posters, pamphlets and charts can be explaine to pregnant women during antenatal visits which lead to a decreased rate of seroconversion.

In addition to this, secondary prevention is by serological screening to identify and treat women who acquire toxoplasma infection during pregnancy. Universal toxoplasma screening for is a debatable issue as even very few developed nations have routine antenatal screening for Toxoplasma gondii by serological tests. In developing countries, this is not feasible due to financial burden and hence, only high risk pregnant women must be screened. We comply to the recommendations of high risk category which includes pregnant women with antenatal history of exposure to possible routes of transmission, those who are immunosuppressed or HIV-positive or those with ultrasound findings such as hydrocephalus, microcephaly, intracranial calcifications, ascites, growth restriction or hepatosplenomegaly. ${ }^{[14]}$ Various serological tests are performed to detect the infection by Toxoplasma IgG and IgM assay. Toxoplasma IgG assay done by Sabin Feldman dye test which is the gold standard test to confirm the infection. Other assay methods include Indirect fluorescent antibody test, Latex agglutination test, Direct agglutination test, Enzyme-linked Immunosorbent Assay and Immunosorbent agglutination assay test. Avidity of $\operatorname{IgG}$ antibodies test helps in differentiating recently acquired infection and infection from distant past. IgM assay is more sensitive and specific in diagnosing acute congenital infection in the fetus and new born. Amplification of B1 gene of T.gondii DNA by Polymerase Chain Reaction is highly sensitive and specific test for diagnosis of congenital, ocular, cerebral and disseminated toxoplasmosis. ${ }^{[1]}$ Emphasis must be on the early first trimester diagnosis of the mother. According to a study, combination of a sensitive test for Toxoplasma specific IgM antibodies and estimation of the avidity of IgG antibodies for $\mathrm{T}$. gondii has the highest predictive value with regard to the time of infection. ${ }^{[15]}$ Congenital toxoplasmosis can be diagnosed in newborn by isolation of $\mathrm{T}$. gondii from the placental or fetal tissue, the umbilical cord, body fluid or infant blood through various tests. Therapeutic treatment of women who acquire toxoplasma infection can reduce the severity of fetal infection. 


\section{Conclusion}

The diagnosis of primary toxoplasmosis in pregnant women early in the first trimester is of utmost importance in order to provide them early therapy or other interventions to prevent congenital toxoplasmosis of foetus which causes NIHF. Follow-up of children with suspected or confirmed infection from birth to adolescence is necessary in order to avoid the hazard and damage from congenital toxoplasmosis. These children need to be followed up for known clinical spectrum and complications of toxoplasmosis later in life by appropriate clinical examination and pathological, biochemical and radiological investigations. There is a need for more complete and accurate population based data regarding incidence of toxoplasmosis and number of cases by mode of transmission. Foetal death due to congenital toxoplasmosis requires detailed autopsy to understand the pathogenesis, newer associations and clinical spectrum of toxoplasmosis.

\section{Acknowledgements}

We thank the patient Department of Obstetrics and Gynaecology, JNMC, Belagavi for the support.

\section{References}

1. Trainor B, Tubman R. The Emerging Pattern of Hydrops Fetalis - Incidence, aetiology and management. Ulster Med J 2006;75(3):185-6.

2. Nagaraja B, Ramana BV, Murty DS, Naidu K, Reddy Kailasanatha B. Prevalence of

3. Toxoplasmosis among Antenatal Women with Bad Obstetric History. IJPRBS, 2012;1(3):222-7.

4. Torgerson PR, Mastroiacovo P. The global burden of congenital toxoplasmosis: a systematic review. Bull World Health Organ 2013;91:501-8.

5. Norton ME, Chauhan SP, Dashe JS. Society for MaternalFetal Medicine(SMFM) Clinical Guideline \#7 : Nonimmune hydrops fetalis. Am J Obstet Gynecol 2015.;127-133.5) Mascaretti RS, Falcão MS , Silva AM, Costa Vaz FA, Leone CR. Characterization of newborns with Nonimmune Hydrops Fetalis admitted to a Neonatal Intensive Care Unit. Rev. Hosp. Clín. Fac. Med. S. Paulo 2003;58(3):125-132.

6. Jones JL, Lopez A,Wilson M, Schulkin J, Gibbs $R$. Congenital toxoplasmosis: a review. Obstet Gynecol Surv 2001;56(5):296-305.

7. Singh S, Munawwar A, Rao S, Mehta S, Hazarika NK. Serologic Prevalence of Toxoplasma gondii in Indian Women of Child Bearing Age and Effects of Social and Environmental Factors. PLoS Negl Trop Dis 2014;8(3):e2737.

8. Singh S, Singh N. Toxoplasmosis is sexually transmitted. Proceeding of IX International Conference on AIDS. Berlin, Germany. 1993; 6-11.

9. Palanisamy M, Madhavan B, Balasundaram MB, Andavar $\mathrm{R}$, Venkatapathy N. Outbreak of ocular toxoplasmosis in Coimbatore,India. Indian J Ophthalmol 2006;54:129-31.

10. Serranti D, Buonsenso D, Valentini P. Congenital toxoplasmosis treatment. Eur Rev for Med Pharmacol Sci 2011;15:193-8.

11. Mittal V, Ichhpujani RL. Toxoplasmosis - An Update. Trop Parasitol 2011; 1(1):9-14.

12. Dubey JP, Beattie CP. Toxoplasmosis of animals and man. Boca Raton, FL: CRC Press, Chapter 1.General Biology: Section V, Epidemiology and Epizootiology. 1988, pp 24.

13. Giannoulis C, Zournatzi B, Giomisi A, Diza E, Tzafettas I. Toxoplasmosis during pregnancy: a case report and review of the literature. Hippokratia 2008;12(3):139-143.

14. Paquet C, Yudin M.H. Toxoplasmosis in Pregnancy: Prevention, Screening and Treatment. J Obstet Gynaecol Can 2013;35:S1-S7.

15. Iqbal J, Khalid N. Detection of acute Toxoplasma gondii infection in early pregnancy by $\mathrm{IgG}$ avidity and PCR analysis. J.Med.Microbiol 2007;(56):1495-9.

*Corresponding author:

Dr Mallikarjun Adiveppa Pattanashetti, Assistant Professor, Department of Pathology, S. Nijalingappa Medical College , Bagalkot, India

Email: mallikarjun2030@gmail.com 